\title{
Electric current arising from unpolarized polyvinyl formal
}

\author{
P K KHARE*, P L JAIN ${ }^{\dagger}$ and R K PANDEY $\mathbf{Y}^{\#}$ \\ Department of Postgraduate Studies and Research in Physics and Electronics, Rani Durgavati University, \\ Jabalpur 482 001, India \\ ${ }^{\dagger}$ Department of Physics, Government P.G. College, Damoh 470 661, India \\ \#Department of Physics, Dr H.S. Gour University, Sagar 470 002, India
}

MS received 29 January 2000; revised 14 August 2000

\begin{abstract}
An appreciable electric current is observed in a system consisting of a polyvinyl formal (PVF) film in a sandwich configuration, in the temperature range $30-110^{\circ} \mathrm{C}$. The maximum value of the current during first heating is found to be of the order of $10^{-10} \mathrm{~A}$ and its thermograms exhibit one transition (i.e. current peak) at around $60^{\circ} \mathrm{C}$. The position of the current peak in thermal spectrum shifts with the heating rate. A temperature dependence of the open circuit voltage is also observed. The activation energy of the process responsible for the current is determined. The magnitude of the current is more in the case of dissimilar electrode systems. It is proposed that the electric current arising from unpolarized metal-polymer-metal system is a water activated phenomenon, which is influenced by the transitional changes of the polymer.
\end{abstract}

Keywords. Electric current; open circuit voltage; water activated phenomenon; plasticization effect.

\section{Introduction}

On heating most polymers (Ieda et al 1975; Radhakrishna and Haridoss 1978; Pillai et al 1979; Shareef et al 1983; Khare and Srivastava 1992; Khare et al 1994) sandwiched between two like or unlike metallic electrodes an electric current, known as short circuit thermally stimulated current (TSC) or spontaneous current (SC), is generated. The current is weak at lower temperatures and increases at higher temperatures. The technique of TSC is now widely employed for obtaining information on the behaviour of trapped charges and the dipole relaxation process in polymeric materials (Turnhout 1975). In the TSC measurements, after some treatments of sample for an introduction of non-thermal equilibrium state, the current which flows during heating is analyzed to reduce either the trapping parameters or the distribution of the relaxation time. Since the various processes apart from the intended one are often superimposed it is necessary for correct interpretation of the TSC data that all possible mechanisms are fully understood and the components due to spurious processes are carefully removed either experimentally or analytically (Sawa et al 1977).

In this paper, the experimental results on TSC of pure polyvinyl formal are discussed to elucidate the mechanism of origin and nature of TSC which may prove to be interesting from both the scientific and technological point of view. The study is also aimed at finding the

*Author for correspondence possibility and existence and nature of the carrier injected from the electrodes and volume generated charge carriers.

\section{Experimental}

Commercial polyvinyl formal (PVF) used in the present investigation, without any further purification/recrystallization was obtained from LOBA Chemical, Bombay. The isothermal immersion technique was utilized for preparing thin film of PVF. PVF has a nonpolar main chain with a weakly polar $\mathrm{CO}$ group rigidly attached to the main chain. The solution was prepared in a glass beaker by dissolving $4.2 \mathrm{~g}$ of PVF in a solvent containing a mixture of benzene and methanol, ratio $3: 2$, at room temperature. The solution was continuously stirred using a teflon-magnetic coated stirrer for $30 \mathrm{~min}$. Thereafter, it was stirred and heated to $40^{\circ} \mathrm{C}$ to yield a homogeneous solution. The glass beaker with the solution was kept in a constant temperature oil bath. The microscopic glass slides, which were ultrasonically cleaned, and vacuummetallized were immersed vertically into the solution. After deposition of the film, the glass slides were taken out and dried in an oven at $40^{\circ} \mathrm{C}$ for $24 \mathrm{~h}$. This was followed by room temperature outgassing at 133.33 $\times 10^{-5} \mathrm{~N} / \mathrm{m}^{2}$ for a further period of $24 \mathrm{~h}$. The aluminium, silver, copper, gold, nickel and zinc metals were vacuumevaporated on the surface of the film to obtain a sandwich configuration. The film thickness was determined capacitively at $50 \mathrm{kHz}$ on the basis of known dielectric constant of PVF (Bandrup and Immergut 1975; Khare 
et al 1999). In the present study care has been taken to avoid currents due to any possible temperature gradient or inherent mechanical stress in the system.

The current was recorded with an electrometer (Keithley 600B) which was carefully shielded and grounded to avoid ground loops or extraneous electrical noise.

\section{Results and discussion}

The spontaneous currents under short circuit condition in an aluminium-polyvinyl formal-aluminium sandwich cell are shown in figure 1 at a linear heating rate of $4^{\circ} \mathrm{C} / \mathrm{min}$. Curves $\mathrm{A}$ and $\mathrm{B}$ correspond to the first and second heating runs respectively. The direction of the current during the first as well as the second heating remains the same. During the first heating, a peak is observed at $60^{\circ} \mathrm{C}$ and then a continuous rise in current is observed. On reheating the sample (curve B), already heated up to $110^{\circ} \mathrm{C}$ in the first run and then allowing it to remain at room temperature overnight, current peak with smaller magnitude compared with the observed current in the first heating run is observed. The successive heating runs at different intervals of time are shown in figure 2 . Curves 1, 2, 3 and 4 correspond to the time intervals of 0 , 5,12 and $18 \mathrm{~h}$ respectively. The current is reproducible in successive heating runs, and the magnitude of the current peak in a particular heating run depends on the time interval between this and the previous heating run, while the specimen was allowed to remain at room temperature

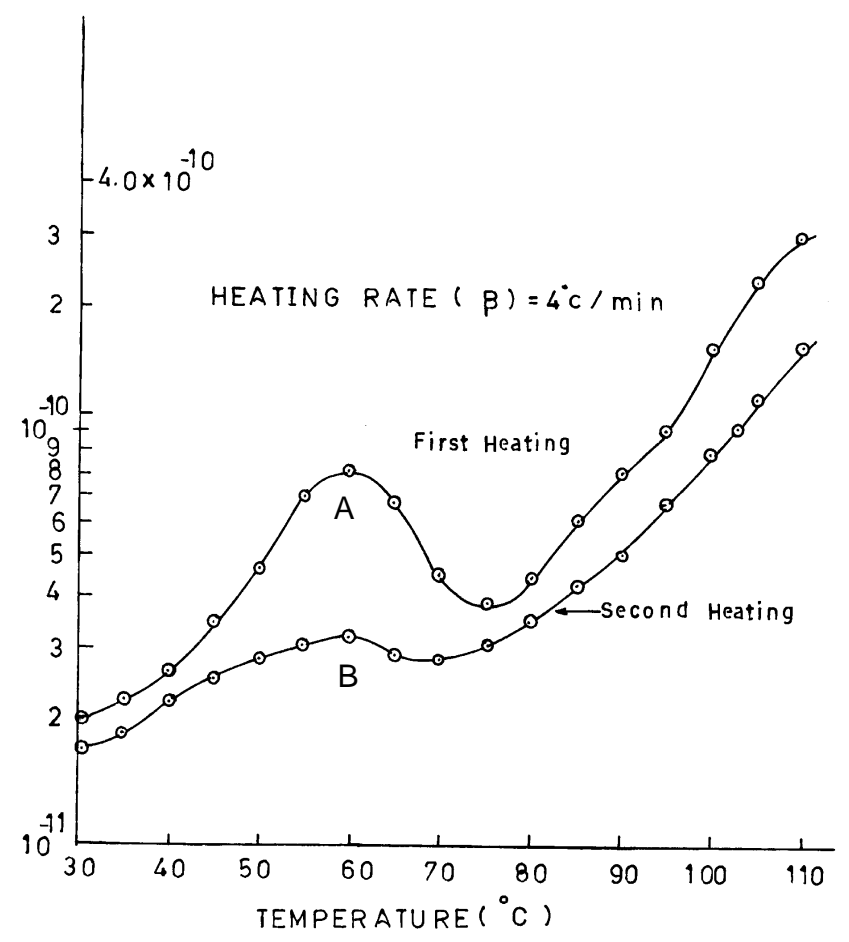

Figure 1. Short circuit vs temperature curves for two heatings (Al-Al electrode system). for this interval of time. If the time interval is short, the magnitude of current peak is also small. Arrhenius plots, i.e. $\log I$ vs $10^{3} / T$ are plotted for the successive heatings of figure 2 (not shown). Though these plots are non-linear, only the straight portion of the plot is used to calculate the activation energy. The activation energy, $U$ of the process responsible for the current has been calculated from the initial current data, using the relation

$$
I \simeq I_{0} \exp (-U / k T)
$$

where $I$ is the observed current, $I_{0}$ a constant, $k$ the Boltzmann's constant and $T$ the temperature. Figure 3 shows the short-circuit currents at three linear heating rates i.e. 2,3 and $4{ }^{\circ} \mathrm{C} / \mathrm{min}$. The effect of decrease in heating rate can be seen in the peak lowering and shifting towards lower temperature side. Short circuit currents obtained from $\mathrm{M}_{1}-\mathrm{PVF}-\mathrm{M}_{2}$ systems, when heated at a uniform heating rate of $4^{\circ} \mathrm{C} / \mathrm{min}$ from room temperature to optimum range of temperature are shown in figure 4. The curves $\mathrm{A}, \mathrm{B}$ and $\mathrm{C}$ correspond to $\mathrm{Cu}-\mathrm{PVF}-\mathrm{Al}, \mathrm{Au}-$ $\mathrm{PVF}-\mathrm{Ag}$ and $\mathrm{Au}-\mathrm{PVF}-\mathrm{Zn}$ systems, respectively. The curves D, E and F correspond to Al-PVF-Zn, Zn-PVF$\mathrm{Zn}$ and $\mathrm{Al}-\mathrm{PVF}-\mathrm{Al}$ systems, respectively. The current for $\mathrm{Cu}-\mathrm{Al}, \mathrm{Au}-\mathrm{Ag}, \mathrm{Au}-\mathrm{Zn}$ and $\mathrm{Al}-\mathrm{Zn}$ electrode systems have been observed in negative direction (i.e. current flows from lower electrode to the upper electrode through the external circuit). The currents in case of similar metallic electrode systems i.e. $\mathrm{Al}-\mathrm{Al}$ and $\mathrm{Zn}-\mathrm{Zn}$ flows from upper to lower electrode direction and has been termed as positive currents. The $\mathrm{Y}$ axis representing the current in positive direction has been shown on right hand side marked as $\mathrm{E}$ and $\mathrm{F}$ on the graph, while that for the nega-

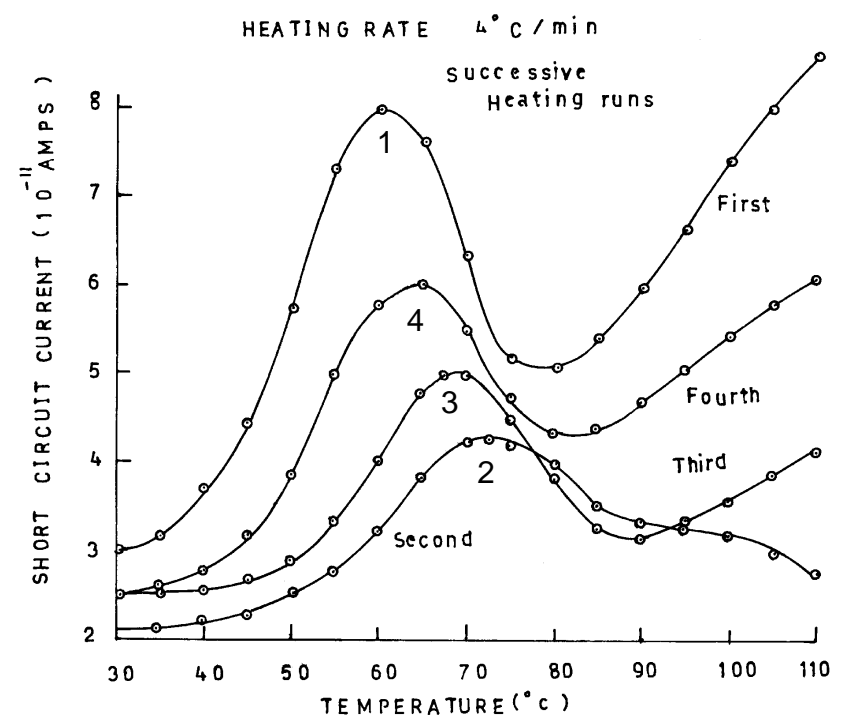

Figure 2. Short circuit current vs temperature plots for successive heatings at a linear heating rate $4^{\circ} \mathrm{C} / \mathrm{min}$ (Al-Al electrode system). 
tive direction has been shown on the left hand side marked as A, B, C and D. The order of current is found to be lower for similar electrode systems than the dissimilar electrode combinations. A current peak is observed in each system, centred around $60^{\circ} \mathrm{C}$. Open circuit voltages have been recorded during the first heating run of a $\mathrm{M}_{1}-\mathrm{PVF}-\mathrm{M}_{2}$ system with a heating rate of $4^{\circ} \mathrm{C} / \mathrm{min}$. Curves A, B, C and D correspond to Al-PVF-Al, Cu$\mathrm{PVF}-\mathrm{Al}, \mathrm{Au}-\mathrm{PVF}-\mathrm{Ag}$ and $\mathrm{Au}-\mathrm{PVF}-\mathrm{Zn}$ respectively (figure 5). Open circuit voltage first rises with temperature exhibiting a broad peak at $55^{\circ} \mathrm{C}$, then it tends to become steady at higher temperatures.

Various interpretations have been advanced for the origin of the short circuit current (Pillai and Mollah 1979a, b; Sharma and Sud 1981; Khare et al 1993). In order to explain the above results we have to consider (i) the generation of charge carriers, and (ii) the electric field driving them. The spontaneous current measurement involves only the thermal treatment of the sample, with zero external field. Charges originate due to thermionic emission from the electrodes and are injected into the dielectric by Richardson-Schottky process. The primary source of charge carriers is the electrode contacts. Charge carriers are generated in the bulk on account of thermal ionization of impurities and absorbed water molecules (Khare et al 1994). Hole injection results in the depletion of the layer of negative charges creating a field at the interface between the electrode and the film surface. We can assume this process to be one of barrier polarization. We can understand our results better if we assume that a system consisting of dielectric sandwiches between electrodes is transferred from an initial state of pure capacitance to a final equilibrium state of pure resistance in series in the presence of charge carriers having different

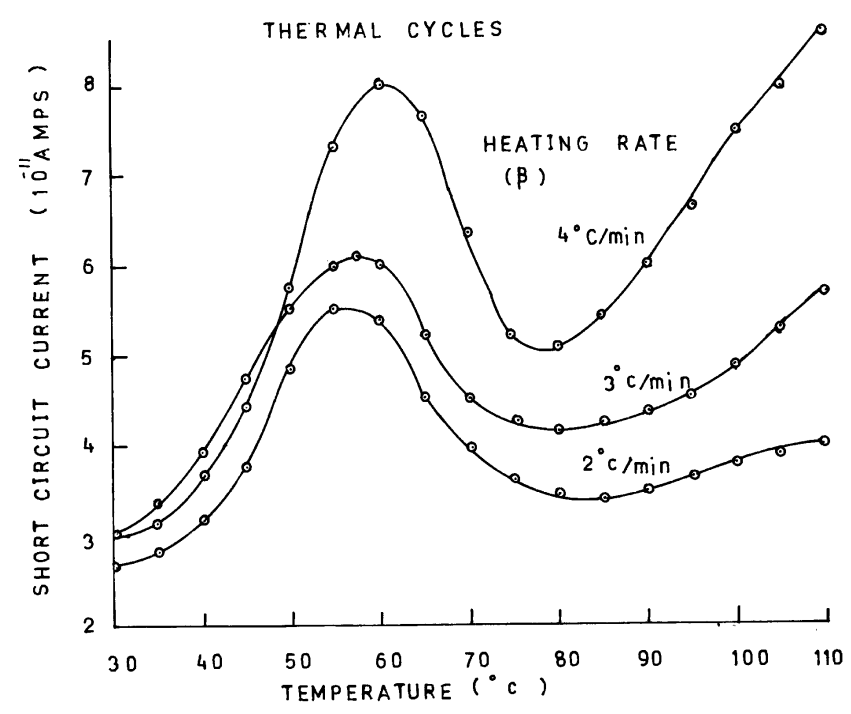

Figure 3. Short circuit current vs temperature plots for different heating rates (i.e. 2,3 and $4^{\circ} \mathrm{C} / \mathrm{min}$ ) ( $\mathrm{Al}-\mathrm{Al}$ electrode system). signs. The resistance of the barrier layer and the bulk material will be determined by the temperature of the system. Charges present in the barrier layers are driven off under the effect of the polarization field developed because of the accumulation of charges in the barrier layers. The magnitude of the observed current in the present study is quite large, so it cannot be due to contact electrification. Further, the current depends on the electrode material used and also on the thermal history of the polymer. Therefore, the observed results may be superposition of the following processes: (i) certain chemical changes such as oxidation, disproportionation, chain splitting, thermal degradation, etc may take place, when a polymer sample, sandwiched between two electrodes is heated. Thereby, a small electrochemical voltage is generated. The current will depend on the magnitude of this voltage and the resistance of the polymer sample, (ii) at low temperatures, the charge carriers within the polymer have a very low mobility, many of them may even be trapped. With rising temperature, the carrier concentration will not increase significantly, but the carrier mobility will increase exponentially. Part of the current may be attributed to stray charges, which may be introduced into the polymer during its fabrication, handling etc and (iii) the third mechanism responsible for current is the

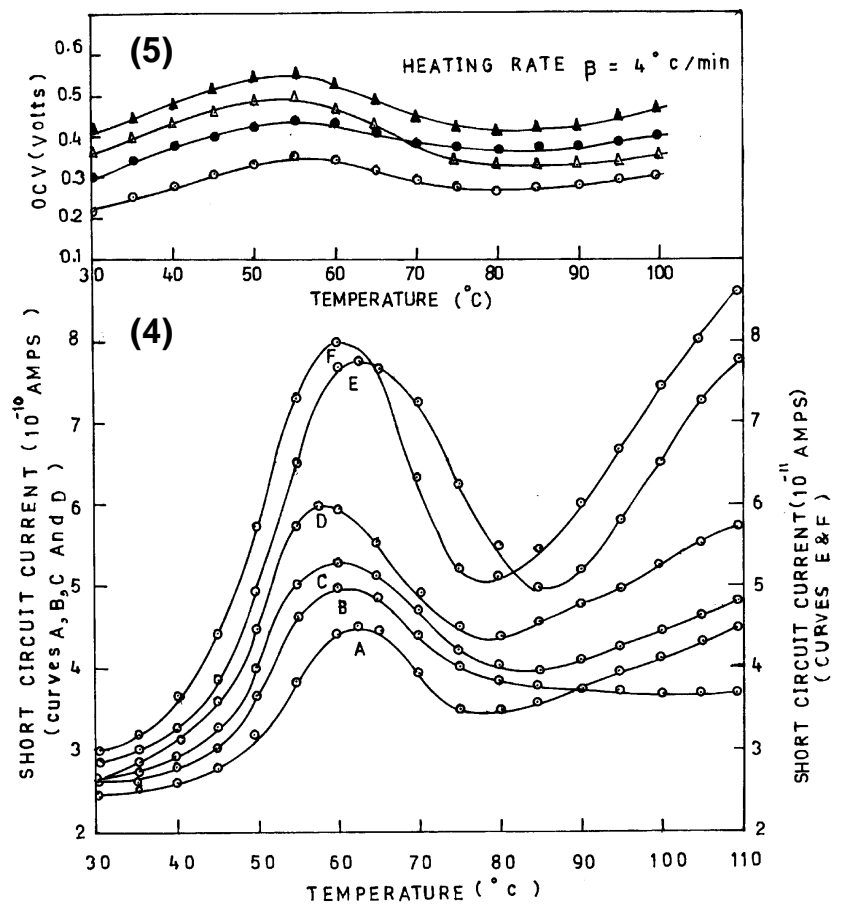

Figures 4-5. 4. Short circuit current vs temperature plots for similar and dissimilar electrode combinations. Curves A, B, C and $\mathrm{D}$ correspond to $\mathrm{Cu}-\mathrm{Al}, \mathrm{Au}-\mathrm{Ag}, \mathrm{Au}-\mathrm{Zn}$ and $\mathrm{Al}-\mathrm{Zn}$ systems, respectively, while curves $\mathrm{E}$ and $\mathrm{F}$ correspond to $\mathrm{Zn}-$ $\mathrm{Zn}$ and $\mathrm{Al}-\mathrm{Al}$ system, respectively and 5. open circuit voltages vs temperature curves. Curves A, B, C and D correspond to $\mathrm{Al}-\mathrm{Al}, \mathrm{Cu}-\mathrm{Al}, \mathrm{Au}-\mathrm{Ag}$ and $\mathrm{Au}-\mathrm{Zn}$ system, respectively. 
disorientation of the permanent dipoles in the polymer (Sharma and Sud 1981).

The spontaneous current emission seems to be an activation-controlled phenomenon, atleast in those dielectrics which contain sorbed water. The sorbed water may be present in various physical states (Murphy 1960; Mikhailov et al 1966) such as loosely and/or strongly bound states, within samples on their surfaces. The desorbed water molecules or a particular fraction of them dissociate into $\mathrm{H}^{+}$and $\mathrm{OH}^{-}$ions under the influence of the voltaic potential difference between the two electrodes. Apparently the water in the samples held in the form of micro impregnations, makes a homogeneous system. All types of electric charge carriers/ions may form an oriented layer on metal surface. Although the exact nature of the activation centres is not clear, the manner in which the water molecules are accommodated in the polymer structure controls the mechanism of the emission process. Grinter and Bowlt (1976) have described that the activation centres at the surface of the specimen are activated by adsorbed water and the centres within the bulk are activated by absorbed water. The heating process could alter the quasi-equilibrium position of an activation centre and could thus lead to the movement of charge carriers. The first heating run empties activation centres and destroy some of them. Removal of water on heating from a virgin hygroscopic sample would increase the resistance of the sample, the consequence of which might be a decrease in current. The current increase due to the transition in the polymer supersedes the current decrease due to the increase in resistance because of water removal (Pillai and Mollah 1979a, b). It has been observed that the appearance of a peak in a particular heating, depends on the time interval. The magnitude of the current is also seen to depend on the time interval. If the time interval is large, the sample absorbs moisture from the air and the water content becomes almost equal to that for a virgin sample. So the shapes of the curves are similar for the first heating and for the $n$th heating which has a large time interval between it and the previous heating. In the second run of heating (figure 2, curve 2) the lowering in peak current and the shifting of peak position towards higher temperatures indicate the reduction of plasticization of water (i.e. absorbed water). On the other hand, the 3rd and 4th heating runs indicate the gradual increase of plasticization effect, i.e. peak current increases and peak temperature shifts towards lower temperature side. The reduction of amplitude of current in successive runs may also be due to the partial melting of crystallites. When the temperature of the sample is lowered, a recrystallization of small crystallites (Takamatsu and Fukuda 1970) occurs. In the process, ion becomes locally trapped and frozen in crystalline regions. When the sample is heated again, the crystallites melt and the ions trapped inside such crystallites are released to flow freely. The early stages of the polymer decomposition involve the development of a strong system of inter-layer crosslinks, immobilizing the initial random orientation of the layers. This cross-linking finally decreases the segmental mobility on cooling; consequently the current amplitude decreases on reheating. The cross-linking reduces or restricts the segmental mobility, while the degradation products lower the glass transition temperature $\left(T_{\mathrm{g}}\right)$ and/or peak temperature $\left(T_{\mathrm{m}}\right)$. Increase in the value of activation energy in the second heating run and then its gradual decrease in the third and fourth runs can also be explained in terms of decrease and then gradual increase in plasticization effect due to moisture content. Figure 3 also shows that current maxima decreased with lower heating rates. The shape and magnitude of the transitional peaks and their location on the temperature scale depend on the heating rate because the polymers change their properties gradually over a certain interval of temperature. A higher heating rate accelerates the dissociation of loosely and strongly bound water. Reorientation of dipoles and rotation of molecular complexes are also accelerated at higher heating rates. Absorbed water molecules may act as a source of ions as plasticizer or as local structure modifier, and also as electron donors (Seanor 1982). However, too high rates are impracticable because the temperature of the sample may lag behind considerably. The slower the heating, the more time the dipoles have to get disoriented and lower the temperature of maxima (figure 3 ). On lowering the heating rate, the first order transition or the temperature where the degradation commences also decreases.

When the same kind of metal was evaporated on both sides of the film, the magnitude of current was relatively small. On the other hand when different kinds of metals were evaporated on each side of the film, the magnitude of current was larger. When two metals are brought in close contact, a potential difference of work function, $\phi_{2}-\phi_{1}$, is created between the two metals. The semiinsulating polymer differs from a metal, however, in that an electric field may exist within the interior of the semiinsulator. For this reason contact potential drop between the metal and semi-insulator may take place within the material rather than at the contact interfaces. Along with the field, there may exist a depletion in the accumulation of charges in the surface layers. The work function of the polymeric material is higher compared to that of the metals used for contacts, which creates an accumulation layer at the interfaces, and leaves charged donors in the bulk. This in turn creates fields at the interfaces of the metal-polymer system. As the sample is heated, the charges may be trapped in the impurity centres and other trapping centres, such as chain coiling (Khare and Srivastava 1992). The net charges injected from the electrodes are predominantly the majority carriers. The charges which essentially give rise to the field at the interfaces are mobilized and the field due to injected charge carriers is weakened, resulting in the release of 
partly compensated bulk field and the motion of charge carriers is determined by the field in the bulk (Khare et al 1994).

The current generated by any MIM system is dependent, to a marked extent, on the reactions at the metalinsulator interface. It is known that if localized states (traps) are distributed throughout an insulator then the band edges are in general severely distorted at the electrode insulator interface (Nadkarni and Simmons 1970). A peculiar effect called the "barrier layer effect" is found at the contact between the metal and insulator. These layers at the junction with metal electrodes or with other insulators when their contact potentials are different, display a sharp change in conductivity. The differences in contact potentials indicate that the binding energies for electrons are different. The electrons pass through such contacts from the side where the energy is high to the side where it is low until that side has acquired charges sufficient to balance out the difference in contact potential (Variyar et al 1982).

The potential developed at an electrode seems to be mixed one comprising of (i) potential difference between the electrodes, (ii) charge exchange between the metal and dielectric due to difference in their work function, and (iii) possible orientation of polar side group along with the water molecule. Vijh (1979) mentioned that the nature of the polymer will influence the measured voltage in two ways: (i) by changing the magnitude of the "series resistance" of the battery, i.e. the different polymers will have different internal resistances of the specimen inspite of equal thicknesses and (ii) by changing the properties of the "electrolyte" (i.e. polymer) of the $\mathrm{M}_{1}-\mathrm{M}_{2}$ battery. The presence of broad peak in OCV vs temperature curves is probably, because of the transitional changes in structure of the polymer. In case of dissimilar electrode combinations, two electrode-dielectric interfaces have electron exchange between them (charged layer) and for these combinations the work function differences of metals may have a controlling nature of OCV in magnitude. The results of OCV can be interpreted in terms of net difference of work function of both the metals $\left(\mathrm{M}_{1}\right.$ and $\mathrm{M}_{2}$ ) with the work function of the polymer (Devies 1969).

The thermally stimulated discharge current (TSDC) (Khare and Chandok 1995) does not exhibit the peak $\left(\sim 60^{\circ} \mathrm{C}\right)$ which is observed in the present study because of (a) the relatively high temperature up to which the electrets are cooled during the formation; (b) desorption of sorbed moisture in keeping the specimen for $180 \mathrm{~min}$ at a constant temperature $\left(60^{\circ} \mathrm{C}\right)$ during the formation and (c) immediate reheating of the specimen which does not permit it to absorb moisture from surroundings. Thus these factors support the presence of the peak at $60^{\circ} \mathrm{C}$ in the present study due to desorption of sorbed moisture.

The generation of short circuit currents and open circuit voltages, however, opens a new area of research, the outcome of which might be helpful for the development of a practically useful dry cell.

\section{References}

Bandrup J and Immergut E H 1975 Polymer handbook (NY: John Wiley) Vol. III

Devies D K 1969 J. Phys. D 21549

Grinter M R and Bowlt C 1976 J. Phys. D 9 L61

Ieda M, Sawa G, Nakamura S and Nishio Y 1975 J. Appl. Phys. 462796

Khare P K and Srivastava A P 1992 Thin Solid Films 208 233

Khare P K and Chandok R S 1995 Polym. Int. 38153

Khare P K, Gour M S and Srivastava A P 1993 Indian J. Pure \& Appl. Phys. 31399

Khare P K, Chandok R S, Dubey N and Srivastava A P 1994 Polym. Int. 35153

Khare P K, Pandey R K and Jain P L 1999 Bull. Mater. Sci. 22 1003

Mikhailov G P, Artyukhov A I and Borisova T I 1966 Polym. Sci. USSR 71383

Murphy E J 1960 J. Phys. Chem. Solids 1151960

Nadkarni G S and Simmons J G 1970 J. Appl. Phys. 41538

Pillai P K C and Mollah M 1979a J. Macromol. Sci. Phys. B16 327

Pillai P K C and Mollah M 1979b J. Appl. Polym. Sci. 23621

Pillai P K C, Goel T C and Xavier S F 1979 Eur. Polym. J. 15 1149

Radhakrishna S and Haridoss S 1978 J. Appl. Phys. 49301

Sawa G, Lee D C and Ieda M 1977 Jpn J. Appl. Phys. 16359

Seanor D A 1982 in Electrical conduction of polymers (NY: Academic Press)

Shareef A U, Saraf K K and Srivastava A P 1983 Phys. Status Solidi 77381

Sharma R and Sud L V 1981 J. Phys. D. Appl. Phys. 141671

Takamatsu T and Fukuda E 1970 Polymer 1101

Turnhout J Van 1975 Thermally stimulated discharge of polymer electrets (Amsterdam: Elsevier)

Vijh A K 1979 J. Appl. Phys. 503764

Variyar M, Khare M L and Bhatnagar C S 1982 Indian J. Pure \& Appl. Phys. 20409 